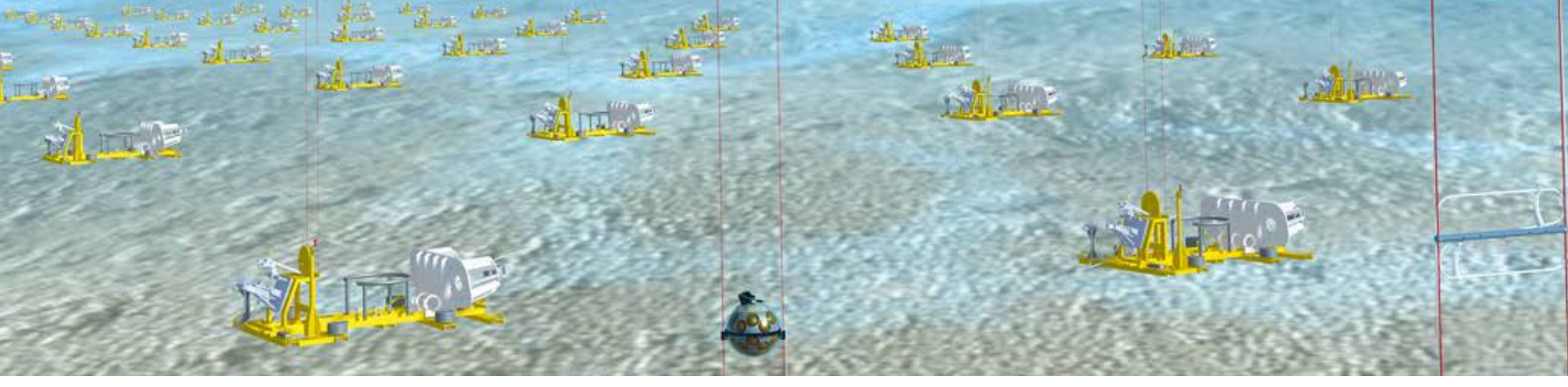

\title{
PROBING THE EARTH'S INTERIOR
}

\section{WITH NEUTRINOS}

\author{
¿ Véronique Van Elewyck ${ }^{1,2}$, João Coelho ${ }^{3}$, Edouard Kaminski ${ }^{4}$ and Lukas Maderer \\ DOI: https://doi.org/10.1051/epn/2021103 \\ ${ }^{1}$ Université de Paris, CNRS, Astroparticule et Cosmologie, F-75013 Paris, France \\ ${ }^{2}$ Institut Universitaire de France, 75005 Paris, France \\ - ${ }^{3}$ Université Paris-Saclay, CNRS/IN2P3, IJCLab, 91405 Orsay, France \\ - ${ }^{4}$ Université de Paris, Institut de physique du globe de Paris, CNRS, F-75005 Paris, France
}

\section{Neutrinos, the lightest entities of the Standard Model of particle physics, can traverse matter like no other known particle. The advent of a new generation of neutrino telescopes is turning these elusive messengers into a new probe to investigate the structure and composition of the deep Earth.}

\section{A virtual journey to the centre of the Earth} Geophysicists have been studying the Earth's interior since the early days of seismology more than a century ago. Seismic waves are a powerful probe that provide information on the Earth internal structures and identify large-scale variations in matter density and properties [1]. Combined with complementary measurements obtained from geodetic techniques, they led to a reference radial model of the Earth density (see Fig. 1).

But the real Earth is more complex, and seismic waves also reveal the presence of large-scale anomalies in the lower mantle, called super-plumes, whose nature, chemistry and origin are still uncertain. They can be remnants of deep primitive material or the product of the progressive recycling of subducted material - two hypotheses with very different consequences for the long-term dynamics of the planet.
Current models also do not univocally constrain the chemical composition of the deep Earth, because of a tradeoff between the temperature and composition effect on geophysical observables. They must rely on indirect information provided by e.g. the study of meteorites or high-temperature/ high-pressure laboratory experiments. All these constraints yield the current consensus of an Iron-Nickel alloy Core containing a few percent of light elements (Silicon, Oxygen, Sulphur, Carbon and/or Hydrogen). The exact nature of the light elements is however not precisely determined yet.

A renewed perspective on this question may come from a completely different branch of physics, dealing with the ghostliest elementary particles: neutrinos. Using these extremely penetrating messengers as a kind of X-ray, a new physical approach to Earth tomography is currently emerging, with the potential to bring original insights about our home planet. $\triangle$ Artist's impression of the KM3NeT neutrino telescope. Credits: KM3NeT. 


\section{A new tool: the neutrino}

In the Standard Model of particle physics, neutrinos are neutral and couple to matter only through the weak interaction, which explains their penetrating power. They exist in three "flavours", each one associated to a companion charged particle: the electron, the muon or the tau. They are produced in a variety of terrestrial and extra-terrestrial sources, with energies ranging from the Mega electron-volt $[\mathrm{MeV}]$ up and above the Peta electron-volt $[\mathrm{PeV}]$.

Since the 80s, neutrino physicists have designed and run a wide range of experiments of ever-increasing dimensions aimed at compensating the small interaction probability of neutrinos with matter. Those patiently accumulated data samples have started unveiling the peculiar fundamental properties of neutrinos [3]. This endeavour recently culminated in the discovery of neutrino flavour oscillations, implying that neutrinos have mass - albeit so tiny that it has not been measured yet. This discovery, awarded the 2015 Nobel Prize in Physics [4], provides the first glimpses into physics beyond the Standard Model and might have far-reaching implications on our understanding of the laws of Nature.

A key contribution came from the study of the abundant and ubiquitous flux of neutrinos originating in the interaction of cosmic rays that continuously hit the Earth's atmosphere and collide with air nuclei. Such collisions produce extended cascades of millions to billions of secondary particles, some of which decay into neutrinos. Most such neutrinos have energies in the range $100 \mathrm{MeV}-10 \mathrm{GeV}$; but their spectrum actually extends up to energies ten to hundred thousand times larger. The probability for a neutrino to interact with ordinary matter is so small that almost all neutrinos created in the atmosphere will simply traverse the Earth and emerge on the other side. A small fraction of them will nevertheless interact and leave a visible signal in one of the neutrino detectors located at the Earth's surface.

\section{Neutrino tomography:} absorption and oscillation

Now, how can these peculiar properties of the neutrino be used for retrieving information about the structure and composition of the Earth [5] ? Neutrino tomography can go along two different paths, depending on the mechanism involved - absorption or oscillation - and on the energy range under study.

At high $(>10 \mathrm{TeV})$ energies, the interaction probability of a neutrino with ordinary matter becomes large enough that absorption effects set in, leading to a progressive attenuation of the flux of through-going atmospheric neutrinos. The Earth becomes completely opaque to neutrinos with $\mathrm{PeV}$-scale energy. The flux attenuation depends on the total amount of matter encountered along the neutrino path and can be inferred from its arrival direction at the detector. The angular distribution of neutrinos detected in different energy ranges will thus provide tomographic information on the matter density inside the Earth.

At lower energies $(1-100 \mathrm{GeV})$, the presence of matter impacts the way neutrinos oscillate from one flavour to another along their path. Ordinary matter contains electrons but no muons nor taus, leading to a net effect on the electron flavour component of the neutrino flux. Depending on the electron density $\mathrm{N}_{e}$, resonance effects may appear, which maximise the probability of oscillation. Typical electron densities inside the Earth will generate such resonances for neutrinos with energies of a few $\mathrm{GeV}$. In this case, the angular and energy distributions of the observed neutrinos inform on the Earth electron content $N_{e}$, which is related to the matter density $\rho_{\mathrm{m}}$ as

$$
\mathrm{N}_{\mathrm{e}}=\left(\mathrm{N}_{\mathrm{A}} / \mathrm{m}_{\mathrm{n}}\right)(\mathrm{Z} / \mathrm{A}) \rho_{\mathrm{m}}
$$

where $\mathrm{N}_{\mathrm{A}}$ is the Avogadro number and $\mathrm{m}_{\mathrm{n}}$ the nucleon mass. The Z/A parameter encodes the ratio of atomic-to-mass

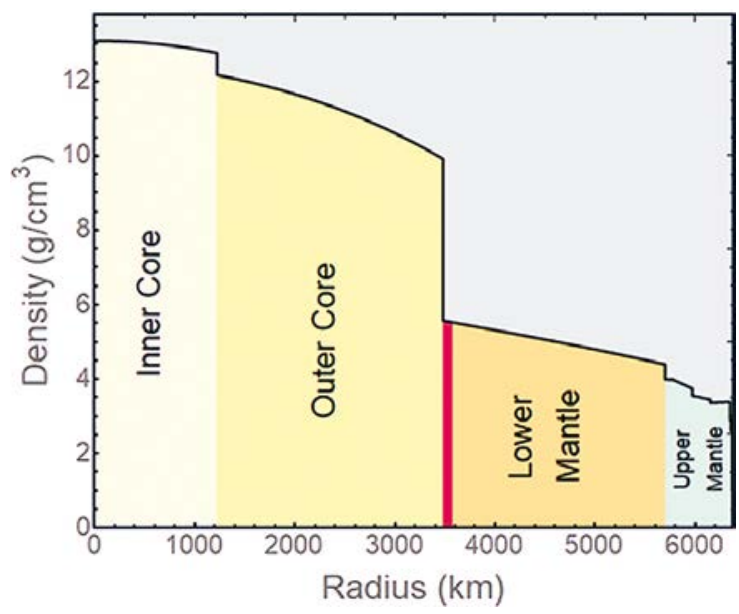

$\triangle$ FIG.1 - Schematic representations of the Earth internal structure (left) showing the main concentric layers and one large-scale structure in the lower mantle known as the African superplume (in red). The right panel shows the density evolution as a function of the radial distance to the Earth center corresponding to the Preliminary Reference Earth Model, PREM [2]. Credits: IPGP \& J. Coelho. 
numbers, which depends on the chemical and isotopic composition of the medium. Assuming a known matter density profile $\rho_{\mathrm{m}}$ (such as the PREM shown in Fig. 1), one can then infer the value of $\mathrm{Z} / \mathrm{A}$ in the distinct Earth layers, that will ultimately translate into compositional constraints. Such an approach can be relevant e.g. to constrain the nature and abundance of light elements in the outer core, in a complementary way to geophysical methods.

\section{Imaging the Earth with neutrino telescopes}

Both approaches to neutrino tomography require large samples of atmospheric neutrinos of different flavours, energies and arrival directions. Physicists therefore rely on Cherenkov telescopes, neutrino detectors that instrument huge volumes of water or ice with photosensors to detect the flash of light that accompanies a neutrino interaction. The IceCube detector [6], that continuously monitors about one cubic kilometer (equivalent to one Gigaton) of South Pole ice, has so far accumulated the largest sample of neutrinos in the $\mathrm{TeV}$ to $\mathrm{PeV}$ range, suitable for absorption tomography studies. Based on a one-year subset of IceCube data, it was possible to reconstruct a rough density profile of the Earth, and to infer its total mass and inertial momentum without relying on any geophysical input [7].

Another large-scale project is under construction on two abyssal sites in the Mediterranean Sea: the cubic-kilometer Neutrino Telescope KM3NeT [8], a distributed infrastructure which uses a novel photosensor design to exploit at best the excellent optical properties of seawater. The KM3NeT detectors consist of arrays of strings anchored to the seabed and supporting pressure-proof glass spheres equipped with multiple photosensors (see introductory illustration). The time and space distributions of the recorded light pulses are used to reconstruct the flavour, energy and direction of the incoming neutrinos. The spatial distribution of photosensors is optimised towards the scientific goal and specific neutrino energy range targeted by each detector. ORCA, off the coast of France, will monitor a few Megatons of seawater, focusing on atmospheric neutrinos in the $1-100 \mathrm{GeV}$ range, with the aim of better understanding their fundamental properties (mass and oscillations). ARCA, close to Sicily, will instrument about 1 Gigaton of water and concentrate on high-energy (TeV-PeV) neutrino astronomy, with a sky coverage complementary to that of IceCube.

By combining the datasets from its two detectors ORCA and ARCA, KM3NeT could become the first neutrino telescope to simultaneously perform oscillation and absorption tomography of the Earth. Preliminary studies give promising perspectives for the determination of the electron density in the inner Earth with ORCA, although the final performance will also depend on the measurement of other parameters, such as the ordering of the neutrino masses (to be determined by ORCA itself and other neutrino experiments on a shorter timescale). Assuming normal mass ordering, after 10 years of operation ORCA will measure the Z/A parameter to a precision of a few percent, both in the mantle and in the core [9]. Such information could help narrow down the allowed parameter space, and lead to new constraints on the primordial Earth composition, the type of convection (thermal vs thermo-chemical), and the long-term evolution of the planet. The stereoscopic combination of data from $\mathrm{KM} 3 \mathrm{NeT}$ and IceCube also provides new perspectives to reconstruct full 3D density and composition profiles, allowing for a better insight into the mantle structure.

One thing is clear to physicists, however: exploiting the full potential of neutrino tomography will require yet another generation of detectors, combining huge instrumented volumes with unprecedented precision in the reconstruction and identification of neutrino events. The challenge of designing a detector fully optimised for Earth tomography might well take another decade; but that will certainly not frighten neutrino physicists who have made theirs the concept of Festina lente.
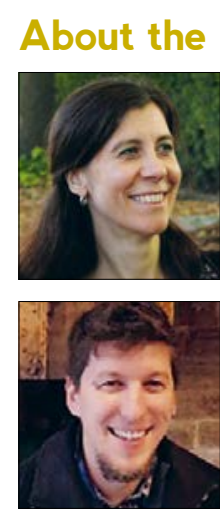

Authors

Véronique Van Elewyck is Assistant Professor at Université de Paris and member of Institut Universitaire de France. She leads the ANTARES/ KM3NeT team at the APC Laboratory. João Coelho, Associate Scientist at the IJC Laboratory, is a neutrino oscillation expert with a focus on atmospheric neutrinos.

Edouard Kaminski is an IPGP Professor of geophysics, specialized in geological fluid dynamics and currently Vice-President for Research at Université de Paris.

Lukas Maderer is a $\mathrm{PhD}$ grantee of the CNRS Interdisciplinary Program 80PRIME. The authors ackowledge the support of LabEx UnivEarthS (ANR-10LABX-0023 and ANR-18-IDEX-0001) for their research.

\section{References}

[1] C. M. R. Fowler, The Solid Earth: An Introduction to Global Geophysics, Cambridge University Press (2004)

[2] A. Dziewonski and D. Anderson, 1981 Physics of the Earth and Planetary Interiors 25, 29 (356)

[3] see https://neutrino-history.in2p3.fr/ for a comprehensive history of neutrino physics

[4] T. Kajita and A. MacDonald - Nobel Lectures 2015, NobelPrize.org

[5] For a conceptual review on neutrino tomography, see W. Winter, Earth Moon Planets 99, 285 (2006)

[6] https://icecube.wisc.edu/

[7] A. Donini, S. Palomares-Ruiz and J.Salvado, NaturePhys. 151, 37 (2019)

[8] http://www.km3net.org

[9] The KM3NeT Collaboration (S. Bourret and V. Van Elewyck), EPJ Web Conf. 207, 04008 (2019) 